\title{
A Rare Case of Primary Pulmonary Anaplastic Large Cell Lymphoma
}

\author{
Joana Carvalho ${ }^{1}$, Diogo Paixão Marques ${ }^{2}$, Inês Oliveira ${ }^{1}$, Cláudia Claudino $^{3}$ \\ ${ }_{1}^{1}$ Pulmonology Department, Hospital de Egas Moniz, Centro Hospitalar de Lisboa Ocidental, Lisbon, Portugal \\ ${ }^{2}$ Internal Medicine Department, Hospital de Egas Moniz, Centro Hospitalar de Lisboa Ocidental, Lisbon, Portugal \\ ${ }^{3}$ Haematology Department, Hospital de São Francisco Xavier, Centro Hospitalar de Lisboa Ocidental, Lisbon, Portugal
}

Doi: 10.12890/2019_001249 - European Journal of Case Reports in Internal Medicine - @ EFIM 2019

Received: 07/08/2019

Accepted: $12 / 08 / 2019$

Published: 09/09/2019

How to cite this article: Carvalho J, Paixão Marques D, Oliveira I, Claudino C. A rare case of primary pulmonary anaplastic large cell lymphoma. EJCRIM 2019;6: doi:10.12890/2019_001249.

Conflicts of Interests: The Authors declare that there are no competing interest

This article is licensed under a Commons Attribution Non-Commercial 4.0 License

\section{ABSTRACT}

Non-Hodgkin lymphomas are rare causes of primary lung neoplasms and most are B-cell in origin. Anaplastic large cell lymphoma is an exceedingly rare type of primary pulmonary lymphoma, with an aggressive clinical course.

We present the case of an 85-year old male patient who attended our Emergency Department complaining of respiratory and constitutional symptoms, and who was found to have a bronchial mass causing subtotal atelectasis of the left lung. Histological examination showed an anaplastic large cell lymphoma and further investigation revealed that it was limited to the lung. To our knowledge, very few similar cases have been reported in the literature.

\section{LEARNING POINTS}

- Non-Hodgkin lymphomas are rare causes of pulmonary lung neoplasms, with the majority of cases being marginal zone B-cell lymphoma of mucosa-associated lymphoid tissue or diffuse large B-cell lymphoma.

- Anaplastic large cell lymphoma (ALCL) usually involves the lymph nodes, skin and soft tissue. It follows an aggressive clinical course and constitutional symptoms are frequent at presentation. Lung involvement may occur as a result of dissemination in up to $12 \%$ of cases. Primary ALCL of the lung is extremely rare.

- Anaplastic lymphoma kinase (ALK) expression is an important prognostic factor, with ALK+ ALCL patients experiencing better outcomes. Adverse prognostic factors also include advanced age, serum lactate dehydrogenase levels and early relapse after therapy.

\section{KEYWORDS}

Anaplastic large cell lymphoma, ALK positive, primary lung lymphoma, lung neoplasm

\section{INTRODUCTION}

Non-Hodgkin lymphoma arising in the lung is rare, accounting for only $0.3 \%$ of primary lung neoplasms ${ }^{[1-3]}$. Marginal zone B-cell lymphoma of mucosa-associated lymphoid tissue (MALT) and diffuse large B-cell lymphoma are responsible for 95\% of all primary pulmonary lymphomas ${ }^{[1-3]}$. Primary anaplastic large cell lymphoma $(A L C L)$ of the lung is an extremely rare type of lung malignancy ${ }^{[1]}$. We report a case of primary pulmonary ALCL presenting with a rapidly growing bronchial mass in an 85-year-old man.

\section{CASE DESCRIPTION}

An 85-year old man presented to our Emergency Department complaining of a several-month history of progressive asthenia, anorexia, productive cough with mucoid sputum and left pleuritic chest pain.

He was a former smoker (10 pack-years) and his medical history included hypertension, hyperlipidaemia and allergic rhinitis, for which he 
was receiving medical treatment. A chest X-ray had been performed 10 days before admission which showed small linear basal opacities, predominantly of the left hemithorax, suggesting mild discoid atelectasis.

On examination, the patient was cyanosed but had a normal respiratory rate without other signs of respiratory distress. His arterial oxygen saturation on room air was $89 \%$; the remaining vital signs were stable. Lung auscultation revealed absent breath sounds in the left hemithorax. There were no other relevant physical findings.

Blood gas analysis of arterial blood drawn while breathing room air showed a $\mathrm{pH}$ of 7.51, a partial pressure of oxygen of $52 \mathrm{mmHg}$, a partial pressure of carbon dioxide of $32 \mathrm{mmHg}$, a serum bicarbonate level of $27 \mathrm{mmol} / \mathrm{l}$ and a serum lactate level of $2.3 \mathrm{mmol} / \mathrm{l}$. Laboratory evaluation revealed raised inflammatory markers (white blood cell count $10.7 \times 10^{\%} / \mathrm{I}$ with a differential of $77 \%$ neutrophils, serum C-reactive protein $6.6 \mathrm{mg} / \mathrm{dl}$ ), elevated D-dimer levels $(1.3 \mu \mathrm{g} / \mathrm{ml})$, impaired renal function (blood urea nitrogen $20 \mathrm{mg} / \mathrm{dl}$, serum creatinine $1.12 \mathrm{mg} /$ dl) and high serum lactate dehydrogenase (LDH) levels (527 IU/I). A new chest X-ray performed in the Emergency Department revealed nearly total opacification of the left hemithorax with left shift of the trachea, suggesting atelectasis (Fig. 1). Contrast-enhanced chest CT was performed, showing a $4.5 \mathrm{~cm}$ bronchial mass causing near-complete occlusion of the distal portion of the left main bronchus with resultant subtotal atelectasis and the fluid bronchogram sign (Fig. 2). The mass also invaded the left lower lobe pulmonary artery where a non-acute pulmonary embolism was observed.

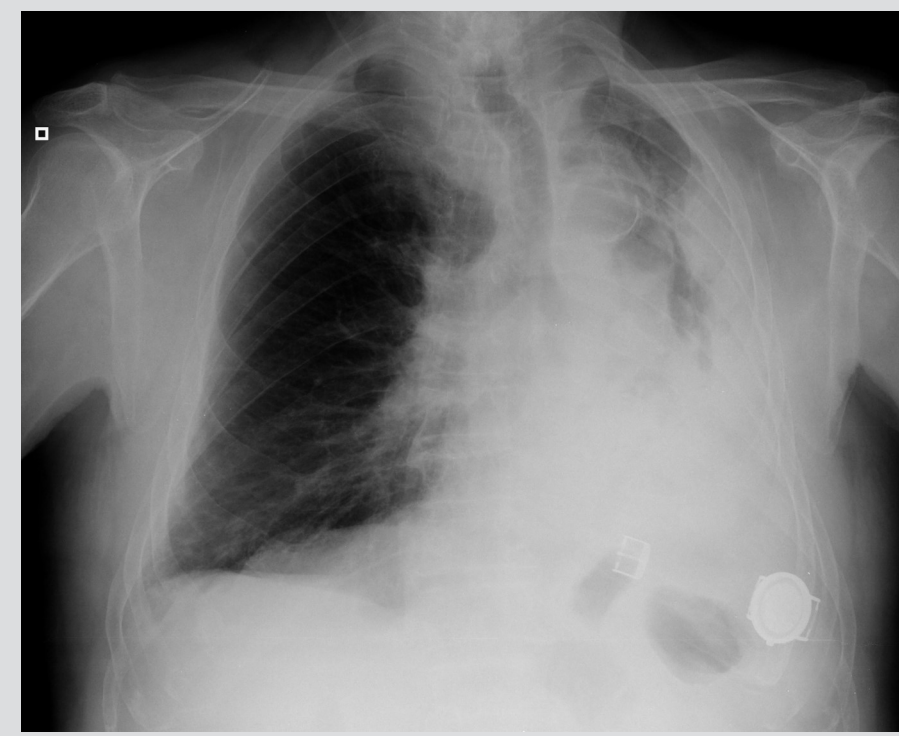

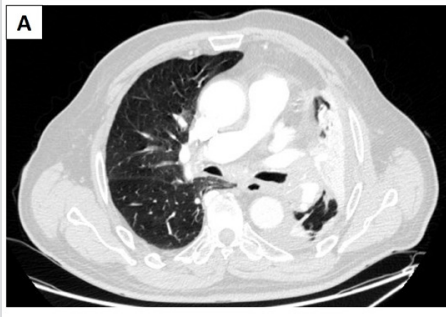
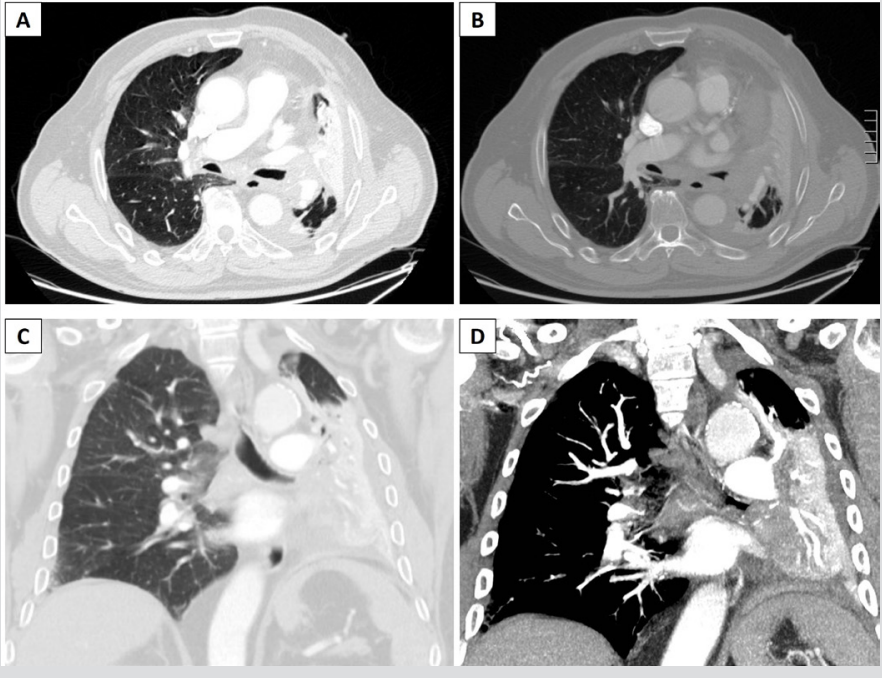

Figure 1. Posterior-anterior chest radiograph at admission, showing nearly total opacification of the left hemithorax with left shift of the trachea, suggestive of atelectasis Figure 2. Contrast-enhanced chest CT at admission, showing a bronchial mass causing near-complete occlusion of the distal portion of the left main bronchus with resultant subtotal atelectasis and a fluid bronchogram sign. There is also invasion of the left lower lobe pulmonary artery. (A, B) Axial and (C, D) coronal views

The patient was admitted to the Pulmonology Department for further investigation of the bronchial mass, as well as treatment of the pulmonary embolism and post-obstructive pneumonia. Empiric antibiotherapy and therapeutic anticoagulation were started.

The bronchoscopy revealed a well-circumscribed and vascularized round-shaped mass located in the distal portion of the left main bronchus (Fig. 3).

\section{DISCUSSION}

ALCL is a peripheral T-cell lymphoma, accounting for about 3\% of adult non-Hodgkin lymphomas and $10-15 \%$ of childhood lymphomas ${ }^{[4]}$.

ALCL cells have unique morphological features, such as an abundant cytoplasm and irregular, eccentric and often horseshoe-shaped nucle ${ }^{[1,3,4]}$. Strong immunoreactivity for CD30 antigen and ALK are also considered diagnostic in this setting ${ }^{[1,3]}$. The most common genetic alteration is a translocation, $\mathrm{t}(2: 5)$ between the ALK gene and the nucleophosmin (NPM) gene ${ }^{[2,4]}$. 


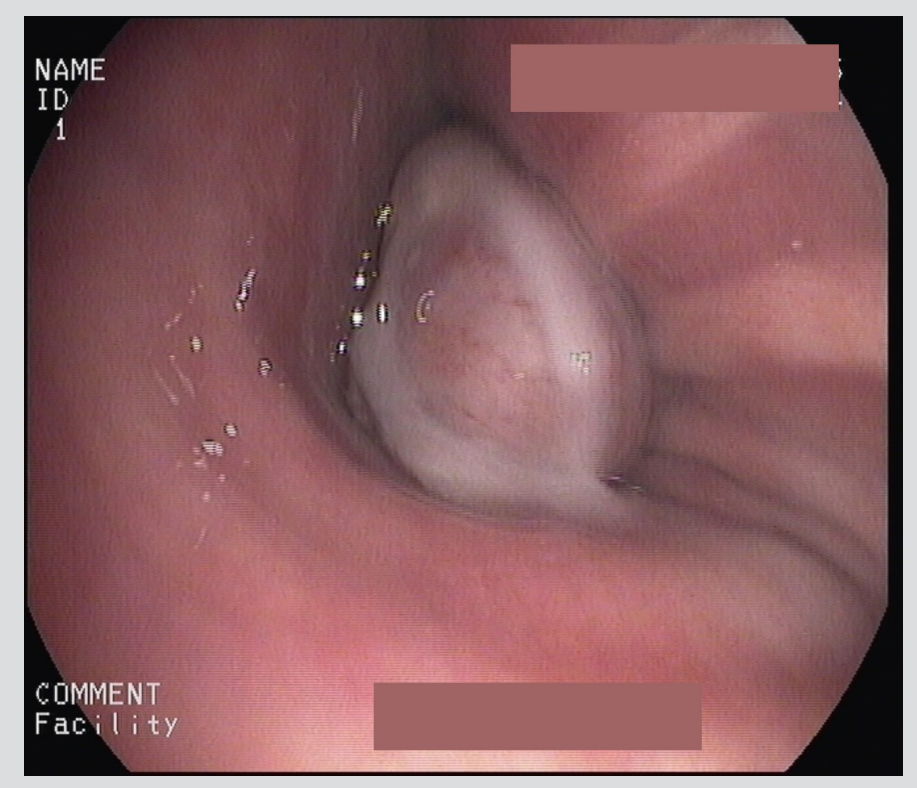

Figure 3. Bronchofibroscopy showing a well-circumscribed and vascularized roundshaped mass located in the distal portion of the left main bronchus

$A L K+A L C L$ is an aggressive lymphoma that occurs more frequently in young subjects, with a male predominance (male to female ratio of $1.5)^{[4]}$. However, it appears to have a bimodal age distribution and can also occur in older adults ${ }^{[5]}$. To the date, no particular risk factor has been identified ${ }^{[4]}$.

ALCL mostly affects lymph nodes, although extranodal involvement can be found in up to $60 \%$ of patients, with the most common sites being the skin, soft tissue and bone ${ }^{[2,4,5]}$. The majority of patients also present with systemic complaints, such as unexplained fever or unintentional weight loss ${ }^{[4]}$. Lung involvement may occur as a result of dissemination in up to $12 \%$ of cases. Primary ALCL of the lung is extremely rare ${ }^{[1,2]}$. To our knowledge, fewer than 20 cases of primary pulmonary ALCL have been reported in the literature ${ }^{[1,3]}$.

Radiological features range from a bronchial mass with the fluid bronchogram sign, as in our patient, to consolidation, ground glass opacities, mediastinal lymphadenopathy, pleural effusion or lung nodules ${ }^{[1]}$. Therefore, the differential diagnosis includes infectious processes, inflammatory or autoimmune diseases and other lung neoplasms ${ }^{[1]}$.

ALK expression is an important prognostic factor, as patients with ALK-negative ALCL tend to have poorer outcomes, with shorter diseasefree and overall survival[ ${ }^{[1-4]}$. Other adverse prognostic factors include advanced age, poor performance status, high serum LDH levels, presence of constitutional symptoms and early relapse after therapy $(<12 \text { months })^{[4]}$.

The standard first-line treatment for $\mathrm{ALK}+\mathrm{ALCL}$ is combination chemotherapy regimens based on anthracycline ${ }^{[3]}$, although other regimens may also be efficacious, with an overall response rate of 90\% [4]. High-dose chemotherapy with autologous stem-cell transplantation is recommended as salvage treatment in young and fit patients with refractory or relapsed ALK+ $\mathrm{LLCL}^{[4]}$.

Unfortunately, our patient died even though he was treated with an appropriate chemotherapy regimen, which was probably due to the presence of numerous adverse prognostic factors, such as advanced age, poor performance status and systemic complaints.

In conclusion, ALCL is an uncommon lung malignancy. Albeit rare, ALCL can mimic metastatic or primary carcinoma and should be considered in the differential diagnosis of large cell neoplasms of the lung, since it requires a specific treatment and has a different prognosis.

\section{REFERENCES}

1. Ende E, Kauffman T, Munoz P, Martinez-Jiménez S. Primary pulmonary anaplastic large cell lymphoma: a rare malignancy and rare cause of the luftsichel sign. Case Rep Radiol 2018; article ID 8574642.

2. Han S, Maeng Y, Kim Y, Jo J, Kwon J, Kim W, et al. Primary anaplastic large cell lymphoma of the lung presenting with acute atelectasis. Thorac Cancer 2014;5(1):78-81.

3. Zhao Q, Liu Y, Chen H, Zhang Y, Du Z, Wang J, et al. Successful chemo-radiotherapy for primary anaplastic large cell lymphoma of the lung: a case report and literature review. Am J Case Rep 2016;17:70-75.

4. Ferreri A, Govi S, Pileri S, Savage K. Anaplastic large cell lymphoma, ALK-positive. Crit Rev Oncol Hematol 2012;82(2012):293-302.

5. Kinney M, Higgins R, Medina E. Anaplastic large cell lymphoma - twenty-five years of discovery. Arch Pathol Lab Med 2011;135:19-43. 